\title{
Equity in utilization of antiretroviral therapy for HIV-infected people in South Africa: a systematic review
}

\author{
Noor Tromp ${ }^{1 *}$, Charlotte Michels ${ }^{1}$, Evelinn Mikkelsen ${ }^{1}$, Jan Hontelez ${ }^{1,2}$ and Rob Baltussen ${ }^{1}$
}

\begin{abstract}
Introduction: About half a million people in South Africa are deprived of antiretroviral therapy (ART), and there is little systematic knowledge on who they are - e.g. by severity of disease, sex, or socio-economic status (SES). We performed a systematic review to determine the current quantitative evidence-base on equity in utilization of ART among HIV-infected people in South Africa.

Method: We conducted a literature search based on the Cochrane guidelines. A study was included if it compared for different groups of HIV infected people (by sex, age, severity of disease, area of living, SES, marital status, ethnicity, religion and/or sexual orientation (i.e. equity criteria)) the number initiating/adhering to ART with the number who did not. We considered ART utilization inequitable for a certain criterion (e.g. sex) if between groups (e.g. men versus women) significant differences were reported in ART initiation/adherence.

Results: Twelve studies met the inclusion criteria. For sex, 2 out of 10 studies that investigated this criterion found that men are less likely than women to utilize ART, while the other 8 found no differences. For age, 4 out of 8 studies found inequities and reported less utilization for younger people. For area of living, 3 out of 4 studies showed that those living in rural areas or certain provinces have less access and 2 out of 6 studies looking at SES found that people with lower SES have less access. One study which looked at the marital status found that those who are married are less likely to utilize ART. For severity of disease, 5 out of 6 studies used more than one outcome measure for disease stage and reported within their study contradicting results. One of the studies reported inconclusive findings for ethnicity and no study had looked at religion and sexual orientation.
\end{abstract}

Conclusion: It seems that men, young people, those living in certain provinces or rural areas, people who are unemployed or with a low educational level, and those being unmarried have less access to ART. As studies stem from different contexts and use different methods conclusions should be taken with caution.

Keywords: Antiretroviral therapy, Equity, South Africa, Systematic review

\section{Introduction}

South Africa is home to the largest HIV-infected population worldwide, with 6.1 million people living with HIV/ AIDS in 2012 [1]. The country also has the largest antiretroviral therapy (ART) program worldwide: with domestic investments amounting to US $\$ 1.9$ billion in 2011 [2], it provided treatment to about $80 \%$ (2.0 million people) of all eligible people in 2012 [1]. Current South

\footnotetext{
* Correspondence: noor.tromp@radboudumc.nl

'Department of Primary and Community Care, Radboudumc, Nijmegen,

The Netherlands

Full list of author information is available at the end of the article
}

African guidelines state that all those with $\mathrm{CD} 4$ cell counts of $\leq 350$ cells $/ \mu \mathrm{L}$ are eligible for ART [3].

Nevertheless, a significant treatment gap of about half a million people remains between those who receive treatment and those in need according to the eligibility criteria [1]. There is little knowledge on which people are deprived from treatment - e.g. by severity of disease, sex, age, socio-economic status (SES) and area of living [4], limiting the development of policy measures to specifically target and improve treatment coverage among these groups. This is illustrated in South Africa's 'National Strategic Plan on HIV, STIs and TB 2012-2016'

\section{Biomed Central}


which flags the importance of inequalities in treatment utilization but is not specific on which marginalized groups should be targeted [5].

It is clear that ART not only improves a patient's health and survival $[6,7]$, but also substantially reduces their infectiousness $[8,9]$. As a result, ART can play an important role in controlling the epidemic in South Africa [10-12]. The World Health Organization (WHO) recently released new consolidated guidelines, taking both the prevention and treatment benefits of ART into account $[13,14]$. The new guidelines state that ART should be provided for HIV infected people with a CD4 cell count of $\leq 500$ cells $/ \mu \mathrm{L}$, who are in a serodiscordant relationship, and/or pregnant [14]. In addition, the WHO also states that guidelines should be expanded when universal access for those with CD4 cell counts of $\leq 350$ cells $/ \mu \mathrm{L}$ has already been achieved [14]. As treatment programs continue to expand, identifying and targeting hard-to-reach populations will be increasingly important.

We determined the current quantitative evidence-base on equity in utilization of antiretroviral therapy (ART) among HIV-infected people in South Africa. This information may provide insight into methods used for equity research and may help policy makers to identify and target hard-to-reach populations.

\section{Methods}

We performed a systematic review on the basis of the Cochrane Handbook for Systematic Reviews of Interventions, Version 5.1.0.4 [15]. Our search was performed on 18 February 2013 using Pubmed, Embase, Central and Psychinfo database. Our search syntax consisted of search terms in four categories (ART, HIV, South Africa and Equity), that were combined using AND. The search strategy is presented in summary in Table 1 and in detail in Additional file 1.

\section{Conceptual model}

Following the WHO's guidance on monitoring equity in AIDS treatment programs [16], we distinguished five domains of coverage: 1) availability of resources; 2) physical and financial accessibility; 3 ) acceptability; 4) use of service; and 5) effective coverage (defined as the proportion of the population in need of an intervention who fully comply with the recommended treatment program). This review focuses on the latter two domains as the other domains feed into these. We included studies on both ART initiation and adherence and this was together labeled as 'ART utilization'. We acknowledge that an individual's health care utilization can be explained by a function of predisposing factors (e.g. education, culture, health beliefs, age and sex), enabling factors (income, health insurance, waiting time, genetic factors) and need factors (perceived need to seek and adhere to care and professional's judgment about people's health status) [17]. We used the terms 'equity' and 'inequity' to reflect differences in utilization of ART by criteria such as severity of disease, age, or SES [18].

\section{Inclusion and exclusion criteria}

A study was included if it: 1) compared for different groups of HIV infected people (by sex, age, severity of disease, area of living, socio-economic status, marital status, ethnicity, religion and/or sexual orientation (i.e. equity criteria (World Health Organization, Guidance on Priority Setting in Health Care (GPS health) in preparation) [19]) the number initiating/adhering to ART with the number who are not); 2) was performed in South Africa; and 3) reported in English. Although some equity criteria are the social determinants of health, severity of

Table 1 Search strategy employed in systematic review of studies on equity in ART utilization in South Africa

\begin{tabular}{|c|c|}
\hline Category & Search terms (in Pubmed database) \\
\hline \multirow[t]{2}{*}{$\overline{A R T}$} & $\begin{array}{l}\text { Antiretroviral therapy, highly active [MeSH Terms] OR ART [title/abstract] OR HAART [title/abstract] OR AR V [title/abstract] } \\
\text { OR ARVs [title/abstract] OR Anti-Retroviral Agents [Mesh] OR antiretroviral [title/abstract] OR anti retroviral [title/abstract] } \\
\text { OR anti-retroviral [title/abstract] OR antiviral [title/abstract] OR therapy [title/abstract] }\end{array}$ \\
\hline & AND \\
\hline \multirow[t]{2}{*}{ HIV } & $\begin{array}{l}\text { Acquired immunodeficiency syndrome [MeSH Terms] OR acquired immunodeficiency syndrome [title/abstract] OR aids } \\
\text { [title/abstract] OR hiv [MeSH Terms] OR hiv [title/abstract] OR human immunodeficiency virus [title/abstract] OR HIV } \\
\text { infections [MeSH Terms] }\end{array}$ \\
\hline & AND \\
\hline \multirow[t]{2}{*}{ South Africa } & (South Africa [MeSH Terms] OR (South [title/abstract] AND Africa* [title/abstract])) \\
\hline & AND \\
\hline Equity & $\begin{array}{l}\text { (Equity [title/abstract] OR equities [title/abstract] OR inequity [title/abstract] OR inequities [title/abstract] OR equality [title/abstract] OR } \\
\text { equalities [title/abstract] OR equal [title/abstract] OR equitable [title/abstract] OR inequality [title/abstract] OR inequalities [title/abstract] } \\
\text { OR unequal [title/abstract] OR disparity [title/abstract] OR disparities [title/abstract] OR vulnerability [title/abstract] OR fairness } \\
\text { [title/abstract] OR unfair [title/abstract] OR social justice [MeSH Terms] OR social justice [title/abstract] OR justice [title/abstract] } \\
\text { OR barrier [title/abstract] OR coverage [title/abstract] OR barriers [title/abstract] OR healthcare disparities [MeSH Terms] OR health } \\
\text { services accessibility [MeSH Terms] OR health services accessibility [title/abstract] OR access to health care [title/abstract]) }\end{array}$ \\
\hline
\end{tabular}


disease is not and therefore we preferred to use the term 'equity criteria' which was put forward by the WHO [World Health Organization, Guidance on Priority Setting in Health Care (GPS health) in preparation] and Tromp et al. [19]. A study was excluded if it: 1) focused on prevention of mother to child transmission (PMTCT), death during follow up, barriers of accessing care or tuberculosis (TB) services for HIV infected patients; 2) was a qualitative study, comment, editorial, economic evaluation or conference abstract; 3) was a duplicate reference from different databases; and 4) reported only differences in groups by a simple comparison with the gross number of people initiating or adhering to ART. We only included studies that take into account the underlying need of a group for ART. For example, the mere fact that more women than men have access to ART does not necessarily indicate an inequity as more women than men may be infected in the country. There was no restriction for publication date for inclusion of studies. Following the Cochrane guidelines grey literature was excluded due to expected low methodological quality of studies [15].

\section{Study selection, data extraction and quality evaluation} Two independent reviewers (CM and EM) assessed if the studies from the database search satisfied the inclusion criteria. First, all studies were screened on the basis of title and abstract, and subsequently on the basis of full-text. Reference lists of the retrieved articles were screened for additional studies (snowballing). The reviewers used a data collection form (Additional file 2) to extract relevant information (study characteristics, results per equity criteria, and study limitations) from the articles. Both reviewers evaluated the quality of studies using a quality-grading protocol (Additional file 2) adapted from existing protocols $[15,20,21]$. The protocol covers 20 indicators and for each item $0-2$ points are given and added up to get an overall quality score (ranging from 0 to 40 points). Studies were categorized as low-quality ( $<20$ points), medium-quality $(20-29)$ or high-quality $(\geq 30)$. During the study selection, data extraction and quality assessment, disagreements were resolved through discussion with a third researcher (NT) until consensus was reached.

\section{Data synthesis and analysis}

A matrix was developed containing the study results per investigated equity criterion. We established the following categories to summarize the results for each equity criteria investigated in a study: 1) associated, differences reported in ART utilization between groups (e.g. men versus women for sex) were significant $(\mathrm{p}<0.05$, or when 1.0 does not fall in $95 \%$ confidence interval $(95 \% \mathrm{CI})$ ); 2 ) not associated differences reported in ART utilization between groups were not significant ( $\mathrm{p}$ value $>0.05$ or 1.0 falls in
95\% CI; contradicting results, within one study contradicting results were reported for differences in ART utilization between groups due to the use of multiple outcome measures for an equity criterion (e.g. CD4 cell count levels and WHO disease stages for the equity criterion severity of disease); and inconclusive results, differences in ART utilization between groups was investigated but the authors drew no conclusions due to small sample sizes.

We adhered to the PRISMA guidelines for reporting of this systematic review [22].

\section{Results}

\section{Study inclusion}

From the initial search (801 articles), 268 studies were duplicates, 483 studies were excluded on the basis of title/abstract and 39 on the basis of full-text screening. Screening of the references of the remaining 11 studies resulted in one extra article and added to a total of 12 studies that are included in this review (Figure 1, Table 2).

\section{Characteristics of included studies}

Seven studies assessed inequities in ART initiation (Table 3) and five studies in ART adherence (Table 4). All studies were based on primary data analysis from observational surveys, except for one study using secondary data [23] and one review [24]. Studies defined ART initiation differently, like 'at least 14 days on ART' [25] or 'visited the ART clinic at least once after testing HIV positive' [26]. Definitions of non-adherence were also varied, and were measured in terms of patients' absence at the clinic for more than one [27] three [28,29] or six [30] months, or in terms of the number of pills not taken and brought back to the clinic (clinic-based pill counts) [31]. The outcome measure used for equity criteria varied widely among studies. For severity of disease, some compared the differences in utilization of ART by WHO disease stages [26-29,31], while others used CD4 cell count levels [26-31] or viral load [28,31]. For age, many different age categories were used. Fatti et al. [29] only included children in the study population, and the oldest age group in that sample is younger than the youngest age group in for example Govindasamy et al. [26] (who compared people below and above 30 years of age). Six studies, all using different databases, investigated urban and rural areas of the Western cape province and two studies reported at the national level [23,24]. More than half of the articles (seven) [25-27,29-32] were of high-quality, three had medium-quality $[28,33,34]$ and two were of low-quality $[23,24]$. Table 5 gives an overview of the quality scoring per study.

\section{Equity in utilization of ART}

For sex, two [24,27] out of ten studies [24-33] that reported on this equity criterion found an association between sex and utilization of ART. In both studies 


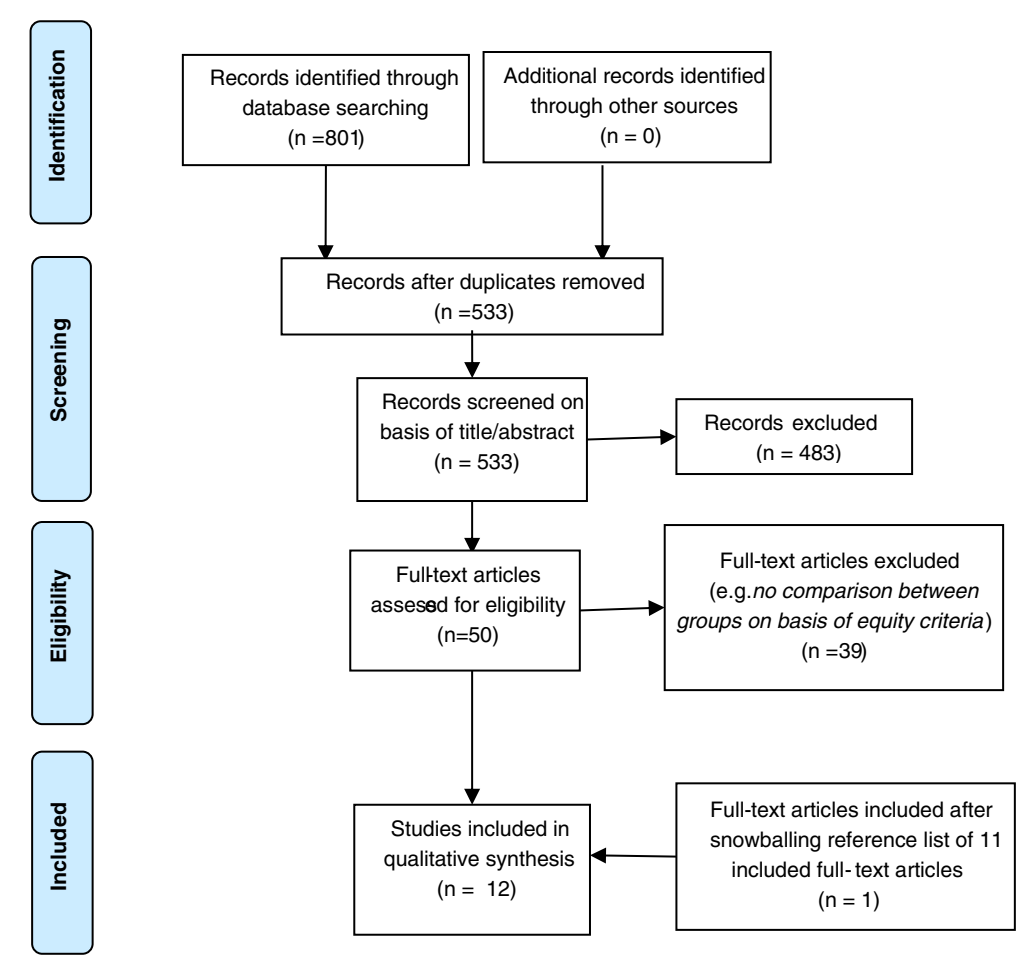

Figure 1 'Flow diagram showing study selection for systematic review of studies on access to antiretroviral therapy in South Africa'.

(high- and low-quality) men appear to have lower utilization of ART compared to women. The other eight studies (six high- and two medium-quality) found no association $[25,26,28-33]$.

Four [30-33] (three high and one medium-quality) out of eight studies [26-33] reported that relatively young people have a lower utilization of ART. The other four studies (three high- and one medium-quality) that reported on age found no association [26-29].

For severity of disease, five [26-29,31] out of six studies [26-31] reported contradicting results. In four [26-28,31] out of these five studies an association was found between ART utilisation and a person's CD4 cell level while no association was found with a patient's WHO status. Of these studies, one ART initiation [26] and one on adherence [27] (both high quality) reported that higher CD4 cell counts are associated with lower utilization of ART. On the contrary, two other studies on adherence (one high- and one medium-quality) reported that lower CD4 cell count is associated with less utilization $[28,31]$. In one other study (high quality) that reported contradicting results for severity of disease among children, an association was found with WHO stage but not with CD4 cell count level [29]. The sixth study (high quality) reporting for severity of disease, only looked at CD4 cell count levels and found that patients with a higher CD4 cell count level adhered less to ART [30].
For area of living, three $[23,29,34]$ out of the four studies $[23,29,32,34]$ that reported on this criterion found an association between area of living and ART utilization. Two studies (high- and medium-quality) reported that people in certain provinces have lower utilization of ART (see Table 3) [23,34]. One of the studies (high-quality) reported that children living in rural areas and who visit ART clinics in urban areas, have lower utilization than children that visit clinics in their own area of living (urban or rural area) [29]. The fourth study (high-quality) that reported on area of living found no association between ART utilization and area of living (peri-urban, urban or rural area) [32].

Socioeconomic status was found to be associated with ART utilization in two $[28,33]$ (both medium-quality) out of the six studies $[25,26,28,31-33]$ that reported on this criterion, which showed that those unemployed have lower utilization of ART. One of these two studies also reported that those with lower education utilize less [33]. Of the four studies that found no association, one (high-quality) found no differences on the basis of employment and education [26]. The other three (all high-quality) found no differences in ART utilization between those with differences in SES [25,31,32]. One of these also found no association between educational level and ART utilization [32].

For marital status only one study (medium-quality) was included in this review and reported that being 
Table 2 Overview of reported findings per study on association between equity criteria and ART initiation or adherence

\begin{tabular}{|c|c|c|c|c|c|c|c|c|}
\hline \multicolumn{2}{|l|}{ Reference } & \multicolumn{7}{|l|}{ Equity criteria } \\
\hline Name, year & $\begin{array}{l}\text { Quality } \\
\text { score }\end{array}$ & Sex & Age & Severity of disease & Area of living & $\begin{array}{l}\text { Socio-economic status } \\
\text { (including education and } \\
\text { employment) }\end{array}$ & Marital status & Ethnicity \\
\hline \multicolumn{9}{|c|}{ ART initiation (lower < higher likelihood to initiate) } \\
\hline $\begin{array}{l}\text { Cleary } \\
2011[25]\end{array}$ & $* * *$ & $\begin{array}{l}\text { Not } \\
\text { associated, } \\
\text { men = } \\
\text { women }\end{array}$ & & & & $\begin{array}{l}\text { SES: not associated, } \\
\text { SES distribution HIV + } \\
\text { in need = ART clinic patients }\end{array}$ & & $\begin{array}{l}\text { Inconclusive results } \\
\text { population size too small } \\
\text { to draw conclusions }\end{array}$ \\
\hline \multirow[t]{2}{*}{$\begin{array}{l}\text { Cooke } \\
2010[32]\end{array}$} & $* * *$ & $\begin{array}{l}\text { Not } \\
\text { associated }\end{array}$ & & & & & & \\
\hline & & $\begin{array}{l}\text { men }= \\
\text { women }\end{array}$ & $\begin{array}{l}\text { Associated, younger } \\
\text { (15-19 yrs), < older } \\
\text { (>19 yrs) }\end{array}$ & & $\begin{array}{l}\text { Not associated, peri- } \\
\text { urban = urban = rural }\end{array}$ & $\begin{array}{l}\text { SES: not associated, index } \\
\text { profile } 1=2=3=4=5(\mathrm{SES}), \\
\text { education: } \text { not associated, } \\
\text { years of education as } \\
\text { continuous variable }\end{array}$ & & \\
\hline $\begin{array}{l}\text { Govindasamy } \\
2011 \text { [26] }\end{array}$ & $* * *$ & $\begin{array}{l}\text { Not } \\
\text { associated, } \\
\text { men = } \\
\text { women }\end{array}$ & $\begin{array}{l}\text { Not associated, } \\
\leq 30 \mathrm{yrs}=\geq 30 \mathrm{yrs}\end{array}$ & $\begin{array}{l}\text { Contradicting results, } \\
\text { CD4 cell count: associated, } \\
\text { CD4 }>350<\leq 350 \\
\text { WHO stages: not associated }\end{array}$ & & $\begin{array}{l}\text { Education: } \text { not associated, } \\
\text { primary school completed }= \\
\text { not completed, employment: } \\
\text { not associated, } \\
\text { employed }<\text { unemployed }\end{array}$ & & \\
\hline $\begin{array}{l}\text { Tsai } \\
2009 \text { [33] }\end{array}$ & $* *$ & $\begin{array}{l}\text { Not } \\
\text { associated, } \\
\text { men = } \\
\text { women }\end{array}$ & $\begin{array}{l}\text { Associated, younger } \\
\text { (18-30 yrs)< older } \\
\text { (30-35 yrs) }\end{array}$ & & & $\begin{array}{l}\text { Education: associated, } \\
\text { lower education } \\
\text { (secondary) < higher } \\
\text { education (matric/ tertiary), } \\
\text { employment: associated, } \\
\text { non salaried employment } \\
\text { < salaried employment, } \\
\text { unemployed < employed }\end{array}$ & $\begin{array}{l}\text { Associated never } \\
\text { married }<\text { married } \\
\text { or cohabiting }\end{array}$ & \\
\hline $\begin{array}{l}\text { Adam } \\
2009 \text { [34] }\end{array}$ & $* *$ & & & & $\begin{array}{l}\text { Associated }{ }^{4} \text {, unequal } \\
\text { ART coverage } \\
\text { between } 9 \text { provinces }\end{array}$ & & & \\
\hline $\begin{array}{l}\text { Muula } \\
2007 \text { [24] }\end{array}$ & * & $\begin{array}{l}\text { Associated }{ }^{4}, \\
\text { male }< \\
\text { female }\end{array}$ & & & & & & \\
\hline $\begin{array}{l}\text { Nattrass } \\
2006[23]\end{array}$ & & & & & $\begin{array}{l}\text { Associated }{ }^{4} \text {, unequal } \\
\text { ART coverage } \\
\text { between } 9 \text { provinces }\end{array}$ & & & \\
\hline \multicolumn{9}{|c|}{ ART adherence (poorer < better adherence) } \\
\hline $\begin{array}{l}\text { Boyles } \\
2011[30]\end{array}$ & $* * *$ & $\begin{array}{l}\text { Not } \\
\text { associated, } \\
\text { men= } \\
\text { women }\end{array}$ & $\begin{array}{l}\text { Associated, younger } \\
(<25 \text { yrs })<\text { older, } \\
(25-50 \text { yrs })\end{array}$ & $\begin{array}{l}\text { Associated, CD4 } \geq 200 \\
<<200\end{array}$ & & & & \\
\hline
\end{tabular}


Table 2 Overview of reported findings per study on association between equity criteria and ART initiation or adherence (Continued)

\begin{tabular}{|c|c|c|c|c|c|c|}
\hline $\begin{array}{l}\text { Orrell } \\
2003[31]\end{array}$ & $* * *$ & $\begin{array}{l}\text { Not } \\
\text { associated, } \\
\text { men = } \\
\text { women }\end{array}$ & $\begin{array}{l}\text { Associated, younger } \\
\text { < older, adherence } \\
\text { group is older ( } 34 \text { yrs) } \\
\text { than non-adhererce } \\
\text { group ( } 31 \text { yrs) }\end{array}$ & $\begin{array}{l}\text { Contradicting results, } \\
\text { CD4 cell count: associated, } \\
\text { patients that not adhere } \\
\text { had lower CD4 level, Viral } \\
\text { load: associated, patients } \\
\text { that not adhere had higher } \\
\text { VL, WHO stage: not associated }\end{array}$ & & $\begin{array}{l}\text { SES: not associated, } \\
\% \text { low SES in patient } \\
\text { group that continued, } \\
\text { ART = that not } \\
\text { continued }\end{array}$ \\
\hline $\begin{array}{l}\text { Kranzer } \\
2010[27]\end{array}$ & $* * *$ & $\begin{array}{l}\text { Associated, } \\
\text { men }< \\
\text { women }\end{array}$ & $\begin{array}{l}\text { Not associated, } \\
\leq 30 \mathrm{yrs}=>30 \mathrm{yrs}\end{array}$ & $\begin{array}{l}\text { Contradicting results, CD4 } \\
\text { cell count: associated, > } 200 \\
<\leq 100, \text { WHO stage: } \\
\text { not associated }\end{array}$ & & \\
\hline $\begin{array}{l}\text { Fatti } \\
2010[29]\end{array}$ & $* * *$ & $\begin{array}{l}\text { Not } \\
\text { associated, } \\
\text { men = } \\
\text { women }\end{array}$ & $\begin{array}{l}\text { Not associated, } \\
\text { younger children } \\
\text { ( } \leq 2 \text { yrs })=\text { older } \\
\text { children ( }>2 \text { yrs })\end{array}$ & $\begin{array}{l}\text { Contradicting results, } \\
\text { CD4 cell count (severe } \\
\left.\text { immunodeficiency }{ }^{1}\right) \text { : } \\
\text { associated, patients } \\
\text { with severe i } \\
\text { mmunodeficiency } \\
\text { were less adherent, } \\
\text { WHO stage (severe } \\
\text { clinical status'): } \\
\text { not associated }\end{array}$ & $\begin{array}{l}\text { Associated, rural/ } \\
\text { urban }<\text { urban/urban } \\
<\text { rural/rural }\end{array}$ & \\
\hline $\begin{array}{l}\text { Cornell } \\
2009[28]\end{array}$ & $* *$ & $\begin{array}{l}\text { Not } \\
\text { associated, } \\
\text { men }= \\
\text { women }\end{array}$ & $\begin{array}{l}\text { Not associated, } \\
\text { age as continuous } \\
\text { variable }\end{array}$ & $\begin{array}{l}\text { Contradicting results, } \\
\text { CD4 cell count: associated, } \\
\text { CD4 }<50<50-150, \text { but CD4 } \\
<50=>150, \text { WHO stage: } \\
\text { not associated, Viral load: } \\
\text { not associated }\end{array}$ & & $\begin{array}{l}\text { Employment: associated, } \\
\text { no income < income }\end{array}$ \\
\hline
\end{tabular}

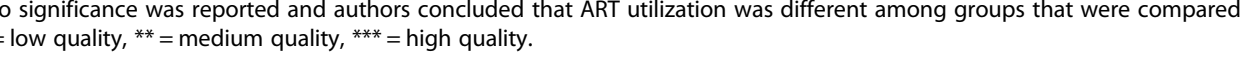


Table 3 Overview of finding per study reporting on equity in ART initiation

\begin{tabular}{|c|c|c|c|c|c|c|}
\hline $\begin{array}{l}\text { Study, quality } \\
\text { score, study } \\
\text { type }\end{array}$ & Study objective & $\begin{array}{l}\text { Study area, type of clinic/ } \\
\text { program }\end{array}$ & $\begin{array}{l}\text { Year of data } \\
\text { collection }\end{array}$ & $\begin{array}{l}\text { Study design (comparison } \\
\text { between population a and b), } \\
\text { population sizes, sampling } \\
\text { method/inclusion criteria }\end{array}$ & $\begin{array}{l}\text { Statistical } \\
\text { analysis }\end{array}$ & $\begin{array}{l}\text { Outcome on association } \\
\text { as reported per equity criteria }\end{array}$ \\
\hline $\begin{array}{l}\text { Cleary } \\
2011{ }^{* * *} \\
\text { Observational } \\
{[25]}\end{array}$ & $\begin{array}{l}\text { To evaluate whether the } \\
\text { distribution of ART services } \\
\text { in the public system reflects } \\
\text { the distribution of people in } \\
\text { need among adults in the } \\
\text { urban population }\end{array}$ & $\begin{array}{l}\text { Urban area: poor } \\
\text { communities in Mitchells } \\
\text { Plain (Cape Town, Western } \\
\text { Cape province) and Soweto } \\
\text { township (Johannesburg, } \\
\text { Gauteng province), public clinics }\end{array}$ & $\begin{array}{l}\text { National } \\
\text { survey: } 2008 . \\
\text { Urban clinic } \\
\text { data: } \\
\text { unknown }\end{array}$ & $\begin{array}{l}\text { a. Population in need for ART } \\
\text { ( } \mathbf{n}=742) \text { : national survey ( } 2008, \\
\text { HIV + residents), sampling unknown, } \\
\text { b. ART patients in urban public } \\
\text { clinics ( } \mathbf{n}=635) \text { : data from ART } \\
\text { users ( }>18 \text { yrs, }>14 \text { days on } \\
\text { treatment) in three clinics in } \\
\text { Mitchells Plain (selected } \\
\text { proportional to the number } \\
\text { of ART patients in facility) and } \\
\text { three in Soweto (stratified } \\
\text { random sampling) }\end{array}$ & $\begin{array}{l}\text { Comparison } \\
\text { distribution of } \\
\text { equity criteria } \\
\text { (i.e. patients } \\
\text { characteristics) }\end{array}$ & $\begin{array}{l}\text { Sex (not associated): percentage of HIV + } \\
\text { women in national survey is same as in } \\
\text { ART users in urban clinic; } 67.4 \% \text { [95\% Cl: } \\
61.5-72.9] \text { versus } 65.7 \% \text { [95\% Cl: } 60.6-70.7] \text {, } \\
\text { p >0.05. Socioeconomic status (not } \\
\text { associated): no significant differences } \\
\text { in SES distribution between HIV + in } \\
\text { need for ART and ART patients in urban } \\
\text { clinics; independence partition Pearson's } \\
\text { chi-square test: } 8 \text { [p }=0.43] \text { Race/ethnicity } \\
\text { (inconclusive results): percentage of } \\
\text { non-African is 2,5\% in population HIV + } \\
\text { in need versus 4.3\% of ART users in } \\
\text { urban clinics, authors state that sample } \\
\text { size of non-African is too small to draw } \\
\text { conclusions on equity }\end{array}$ \\
\hline $\begin{array}{l}\text { Cooke } \\
2010 * * * \\
\text { Observational } \\
{[32]}\end{array}$ & $\begin{array}{l}\text { To investigate factors } \\
\text { associated with uptake } \\
\text { of ART through a primary } \\
\text { health care system in rural } \\
\text { South Africa }\end{array}$ & $\begin{array}{l}\text { Rural, peri-urban and urban } \\
\text { areas: Hlabisa sub-district, } \\
\text { Umkhanyakude district, Northern } \\
\text { KwaZulu-Natal province, public } \\
\text { clinics supported by NGOs }\end{array}$ & $\begin{array}{l}\text { Aug } 2004- \\
\text { Dec } 2008\end{array}$ & $\begin{array}{l}\text { a. HIV + residents not on ART } \\
(\mathbf{n}=\mathbf{1 , 0 0 3 )} \text { : population-based } \\
\text { surveillance in } 6 \text { catchment areas, } \\
\text { b. HIV + residents on ART } \\
(\mathbf{n}=\mathbf{1 , 2 5 1 )} \text { : population based } 2008 \\
\text { cohort (HIV+, > } 15 \text { yrs, on ART) }\end{array}$ & $\begin{array}{l}\text { Multivariate } \\
\text { logistic } \\
\text { regression }\end{array}$ & 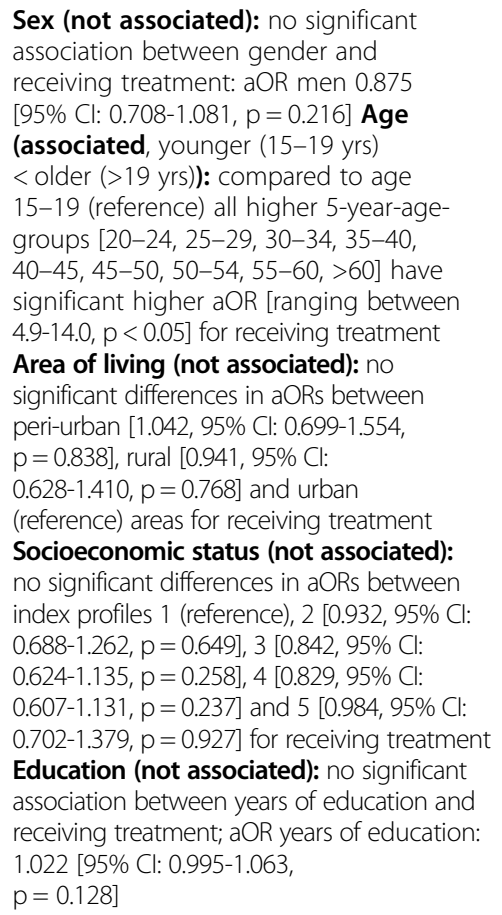 \\
\hline
\end{tabular}

$\begin{array}{ll}\mathbf{2 0 1 0} * * * & \text { associated with uptake } \\ \text { Observational } & \text { of ART through a primary } \\ \text { [32] } & \text { health care system in rural }\end{array}$

South Africa areas: Hlabisa sub-district,

mkhanyakude district, Northern

KwaZulu-Natal province, public

clinics supported by NGO $(\mathbf{n}=\mathbf{1}, \mathbf{2 5 1})$ : population based 2008

$p=0.128$ 
Table 3 Overview of finding per study reporting on equity in ART initiation (Continued)

\begin{tabular}{|c|c|c|c|c|}
\hline $\begin{array}{l}\text { Govindasamy } \\
2011^{* * *} \\
\text { Observational } \\
{[26]}\end{array}$ & $\begin{array}{l}\text { To assess the proportion and } \\
\text { characteristics of individuals } \\
\text { who accessed HIV care after } \\
\text { testing HIV + in a mobile } \\
\text { testing unit }\end{array}$ & $\begin{array}{l}\text { Rural area: Cape Metropolitan } \\
\text { region, Western Cape province, } \\
\text { type of clinic not clearly } \\
\text { reported }\end{array}$ & $\begin{array}{l}\text { Tested HIV+: } \\
\text { 2008-2009. } \\
\text { Interviewed: } \\
\text { Apr-Jun } \\
2010 .\end{array}$ & $\begin{array}{l}\text { Patients tested HIV + in mobile } \\
\text { testing units that: a. linked to } \\
\text { ART care (i.e. receiving CD4 } \\
\text { test result), b. not linked, } \\
\text { A random sample of patients } \\
\text { tested HIV + between August } \\
2008 \text { - December } 2009 \text {, } \\
\geq 18 \text { yrs, CD4 }<350, \text { received } \\
\text { CD4 test results, available } \\
\text { socio-demographic variables } \\
\text { was selected using mobile } \\
\text { testing unit records }(\mathbf{n}=\mathbf{7 7})\end{array}$ \\
\hline
\end{tabular}

Binomial

univariate

bivariate

regression

analysis

Tsai $2009 * *$

Observational

[33]
Rural area: Limpopo

province, public hospital
To assess differences in socioeconomic profiles between those who access HIV-related clinical services and the HIV-infected individuals living in the wider community
Community survey: 20042005. Clinic 2005. Clinic survey: Jan $2003-$
( $\mathrm{n}=242)$ : household

survey, random sampled

from eight rural villages in

the province (14-35 yrs, HIV+),

b. clinical sample, HIV + on

ART $(n=534)$ : convenience

sample of patients (18-35 yrs)

in primary HIV/AIDS provider

hospital, referred by 45 primary

hoalth care clinics. Note: samples

were not taken from identical

sub-districts

Sex (not associated): same likelihood to link to care for female as male patients; **RR female: 1.18 [95\% Cl. 081-1.72

$\mathrm{p}=$ not reported, 1.0 falls within $\mathrm{Cl}$ ] Age (not associated): same likelihood to link to care for younger ( $\leq 30$ years) as older patients ( $\geq 30$ years) to link to care; **RR $\geq 30$ years: 1.21 [95\% Cl: 0.83-1.77, $p=$ not reported, 1.0 falls within $\mathrm{Cl}$ S Severity of disease (contradicting results, CD4 cell count associated and WHO stages not associated): significantly lower likelihood to link to care for patients with high (>350) compared to low $(\leq 350)$ CD4 cell count; ${ }^{*} R R$ CD4 > 350: 0.49 [95\% Cl: 0.27-0.87, $p=0.014] /$ same likelihood to link to care for patients in WHO stage I as WHO stage II, III or IV; **RR WHO clinical stage I: 0.88 [95\% Cl: 0.65-1.18, p = not reported, 1.0 in Cl]

Education (not associated): same likelihood to link to care for patient completed primary school as patients that have not; **RR completed primary school: 1.17 [95\% Cl: $0.66-2.08, p=$ not reported, 1.0 falls within $\mathrm{Cl}$ Employment (not associated employed < unemployed): likely lower likelihood to link to care for employed compared to unemployed patients; **RR employed: 0.72 [95\% Cl: 0.51-1.01, $p=0.056$ ]. ${ }^{*}=$ univariate $^{* *}=$ bivariate analysis

Uni-variate comparison regression Sex (not associated): no significant difference percentage women in the vs. $79 \%$ [p = 0.78] Age (associated, younger (18-30 yrs) < older (30-35 yrs)): significant difference in age distribution between community and clinic sample: 18-20 yrs: $13 \%$ vs 3.6\%; $21-25$ yrs: $33 \%$ vs. $16 \%$; $26-30 \mathrm{yrs}: 36 \%$ vs $33 \%$; $31-35$ yrs: $18 \%$ vs. $47 \%$; $X^{2}=85$ [p $\left.<0.001^{*}\right]$ Education (associated, higher education > lower education): significant difference in distribution educational attainment between community and clinic attainment between community and clin
sample: in clinic less likely to completed secondary education $[p<0.001]$, but more likely to completed matric or tertiary education $[p=0.04] X^{2} 42\left[p<0.001^{*}\right]$ Employment (associated, not having salaried employment $<$ having salaried employment, unemployed < employed): 


\author{
Adam $2009 * *$ To quantify the coverage \\ Rural and urban: National/ \\ Observational in South Africa up to the middle nine provinces, public clinics \\ [34] \\ of 2008 , according to various
definitions of antiretroviral \\ treatment eligibility
}

Muula 2007 * To describe the gender

Systematic distribution of patients

review [24] distribution of patients Southern Africa

Nattrass 2006* To compare ART roll-out

Critical in public sector betwee
Rural and urban: National

(1999-2004), Khayelisha township

in Capetown (2001-2), Eastern

cape province 2001-4), Northern

cape province

(2001-5), public clinics

Rural and urban: National

(nine provinces), public clinics
2008

For nine provinces: $\mathbf{a}$. number of HIV + in need for ART: Markov model on HIV progression using different CD4 count compartments b. number of HIV + on ART: estimates of patients starting ART in public health facilities using

Department of Health unpublished internal report (7 May 2009)

$2000-2006$ a. National HIV + prevalence female/male ratio in 2005 b. access to ART female/male ratio. Sampling methods not reported

\section{3 - 2005 For nine provinces:}

\section{a. number of HIV + in need}

\section{for ART,}

b. number of HIV + on ART,

estimates of ART coverage based

on ASSA2003 demographic model

(includes public NGOs and private

sector providers) significant difference percentage having salaried employment between community and clinic sample: $6.2 \%$ vs. $11 \%, X^{2} 3.8$ $[\mathrm{p}=0.05]$ and in percentage unemployed and able to work: $57 \%$ vs. $37 \% ; X^{2} 26$ and able to work: $57 \%$ vs. $37 \% ; x^{2} 26$
$\left[p<0.001^{*}\right]$ Marital status (associated never married < married or cohabiting): significant difference distribution marital status between community and clinic sample: never married: $78 \%$ vs. $43 \%$; married/ cohabiting: $16 \%$ vs. $30 \% ; x^{2}$ $83\left[\mathrm{p}<0.001^{*}\right]^{*}$ also significant after multivariable regression

\section{Comparison Area of living (associated, unequal} ART coverage coverage among nine provinces): unequal ART coverage in 2008 among 9 provinces: Eastern Cape $32.4 \%$, Free State $25.8 \%$ Gauteng 43.5\%, KwaZulu-Natal 39.4\%, Limpopo 32.2\%, Mpumalanga 31.2\%, Northern Cape 61.1\%, North West 35.4\%, Western Cape $71.1 \%$

Comparison Sex (associated, male < female): female have higher access than men to ART: HIV prevalence female/male ratio $=1.2$, while 4 studies report access to ART female/male ratio of $1.9,2.3,1.8$ and 1.5

Comparison Area of living (associated, unequal ART coverage coverage among 9 provinces): unequal data $\quad$ ART coverage at the end of 2005 among 9 provinces: Eastern Cape 21.8\%, Free State 21.0\%, Gauteng 29.6\%, KwaZulu-Natal 20.0\%, Limpopo 27.3\%, Mpumalanga 20.9\% Northern Cape $32.3 \%$, North West 24.5\%, Western Cape $55.7 \%$ 
Table 4 Overview of findings per study reporting on equity in ART adherence

\begin{tabular}{|c|c|c|c|c|c|c|}
\hline $\begin{array}{l}\text { Study, quality } \\
\text { score, study } \\
\text { type }\end{array}$ & Study objective & $\begin{array}{l}\text { Study area, type of } \\
\text { clinic/program }\end{array}$ & $\begin{array}{l}\text { Year of } \\
\text { data } \\
\text { collection }\end{array}$ & $\begin{array}{l}\text { Study design (comparison between } \\
\text { population a and b), population } \\
\text { sizes, sampling method and } \\
\text { inclusion criteria }\end{array}$ & $\begin{array}{l}\text { Statistical } \\
\text { analysis }\end{array}$ & $\begin{array}{l}\text { Main outcome of analyzed equity } \\
\text { criteria }\end{array}$ \\
\hline $\begin{array}{l}\text { Boyles } 20111^{* * *} \\
\text { Observational } \\
\text { [30] }\end{array}$ & $\begin{array}{l}\text { To determine the factors predicting } \\
\text { loss to follow-up and mortality in a } \\
\text { public-sector HIV and ART } \\
\text { programme in rural South Africa }\end{array}$ & $\begin{array}{l}\text { Rural area: Elliotdale/ } \\
\text { Xora area of Mbhashe } \\
\text { sub-district in Eastern } \\
\text { Cape province, } \\
\text { combined public/donor } \\
\text { program }\end{array}$ & $\begin{array}{l}\text { Jan } 2005- \\
\text { Sept } 2009\end{array}$ & $\begin{array}{l}\text { a. HIV + patients that loss to } \\
\text { follow up }(\mathbf{n}=\mathbf{1 1 7}(6.5 \%)) \text {, } \\
\text { b. HIV + patients that do not loss } \\
\text { to follow up }(\mathbf{n}=\mathbf{1 6 8 6 ) .} \text {. Both } \\
\text { groups are patients enrolled in } \\
\text { clinics of Madwaleni HIV wellness } \\
\text { and ART program including } \\
\text { adherence counseling and home } \\
\text { visits (i.e. Madwaleni Hospital, } \\
\text { its } 7 \text { primary healthcare feeder } \\
\text { clinics and a community based } \\
\text { outreach program): tested HIV+, } \\
\text { ART naïve at time of study } \\
\text { enrollment, }>19 \text { years, initiated } \\
\text { ART (CD4 < } 200 \text { CD4), could be } \\
\text { follow for at least } 3 \text { months }\end{array}$ & $\begin{array}{l}\text { Multiple Cox } \\
\text { proportional } \\
\text { hazard } \\
\text { regression }\end{array}$ & $\begin{array}{l}\text { Sex (not associated): females and males } \\
\text { have same risk of being loss-to-follow-up: } \\
\text { HR female: } 1.42 \text { [95\% Cl 0.90-2.23, } \\
p=0.134] \text { Age (associated, younger } \\
\text { (<25 yrs) < older ( } 25-50 \text { yrs)): younger } \\
\text { people have significant higher risk } \\
\text { to loss-to-follow-up: HR <25 yrs (compared } \\
\text { to } 25-50 \text { yrs): } 1.87 \text { [95\% Cl: } 1.15-3.05, \\
p=0.012] \text { Severity of disease (associated } \\
\geq 200 \text { CD4<<200 CD4): higher CD4 cell } \\
\text { count significantly increases risk to } \\
\text { loss-to-follow-up: } 50-199 \text { CD4 (referent); } \\
\text { HR 0-49 CD4: } 1.00 \text { [95\% Cl: } 0.61-1.64, \\
p=0.019] ; H R \geq 200 \text { CD4: } 1.74 \text { [95\% Cl } \\
1.09-2.78, p=0.019]\end{array}$ \\
\hline
\end{tabular}

Orrell $2003 * * *$

Observational

[31]
To determine adherence of an indigent African HIV-infected cohort initiating ART to identify predictors of incomplete adherence and virologic failure
Urban area: Cape Town, Jan 1996 - a. Patients discontinued Western Cape province, May $2001 \mathbf{4 8}$ weeks of ART $(\mathbf{n}=\mathbf{4 7})$, university of Cape Town clinic

b. Patients that completed

48 weeks of ART $(n=242)$ Both groups are from Cape Town AIDS Cohort (CTAC): HIV + patients, presenting at University of Cape Town HIV clinics (referred by health care workers in the public sector of the wider Cape town area, mainly serving indigent populations), were ART naïve and eligible for adherence monitoring
T-test (age, VL, Sex (not associated): no significant CD4 cell difference in percentage female between count), $X^{2}$ test those discontinued (40.4\%) and completed (gender, (43.4\%) 48 weeks of ART [p=0.7] Age socioechomic (associated, younger < older): those status) discontinued ART before 48 weeks were significantly younger (31 yrs) than those completed (34.1 yrs) $[\mathrm{p}<0.005]$ Severity of disease (contradicting results, CD4 cell count associated and WHO stages not associated): those discontinued ART before 48 weeks had significantly lower mean CD4 cell count (197) than those completed (268) $[p<0.01]$ / those discontinued before 48 weeks ART had a significantly higher VL (5.71 $\log _{10}$ ) than those completed (5.49 $\left.\log _{10}\right)[p<0.05] /$ no significant difference in percentage WHO stage 3 or 4 between those discontinued (49.2\%) and completed (38.2\%) 48 weeks of ART $[p=0.2]$ Socioeconomic status (not associated): no significant difference in the percentage of patients with low socio-economic status in the group that discontinued (36.2\%) and completed (43.6\%) 48 weeks of ART $[p=0.4]$ 
Table 4 Overview of findings per study reporting on equity in ART adherence (Continued)

\begin{tabular}{|c|c|c|c|c|}
\hline $\begin{array}{l}\text { Kranzer } \\
2010 * * * \\
\text { Observational [27] }\end{array}$ & $\begin{array}{l}\text { To investigate the frequency and } \\
\text { risk factors of defaulting treatment } \\
\text { and identify factors associated with } \\
\text { subsequent return to care in a } \\
\text { long-term treatment cohort in } \\
\text { South Africa }\end{array}$ & $\begin{array}{l}\text { Peri-urban: township in } \\
\text { Cape Town, Western } \\
\text { Cape province, public } \\
\text { clinic }\end{array}$ & $\begin{array}{l}\text { Mar } 2004 \text { - } \\
\text { Dec } 2009\end{array}$ & $\begin{array}{l}\text { a. HIV + patients that defaulted } \\
\text { ART }(\mathbf{n}=\mathbf{2 9 1}) \text {, } \\
\text { b. HIV + patients that not } \\
\text { defaulted ART ( } \mathbf{n}=\mathbf{8 6 3}) \text {. Both } \\
\text { groups are from patients presenting } \\
\text { at public-sector primary care clinic } \\
\text { (single ART server in the area), } \\
>15 \text { years, started ART (until } \\
2007<350 \text { CD4 cells (NIH } \\
\text { research study), after } 2007 \\
<200 \text { CD4 cells (provincial } \\
\text { ART program) ( } \mathbf{n = 1 1 5 4 )}\end{array}$ \\
\hline
\end{tabular}

Fatti

$2010 * * *$

Retrospective

cohort study [29]

To compare clinical, immunological and virological outcomes between rural and urban children on ART in large cohort from multiple public health facilities in four provinces of South Africa
Rural and urban: areas in Western Cape,

KwaZulu-Natal, Eastern Cape and Mpumalanga province, public clinics supported by NGOs
Nov 2003 - a. Children on ART that loss to Mar 2008 follow up $(\mathbf{n}=179)$,

\section{b. Children on ART that do}

not loss to follow up ( $\mathrm{n}=$

2153). Both from retrospective

cohort of children, (<16 yrs, ART

naïve), enrolled in 44 routine

public healthcare facilities

(7 rural, 33 urban/12 secondary

level hospitals, 32 primary health

care clinics) supported by a NGO,

used electronic data collection

systems fo

Children were divided in

3 groups a) urban residence

and urban ART facility attended

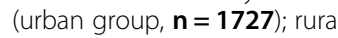

residence and rural facility

attended (rural group, $\mathbf{n}=\mathbf{2 2 8}$ );

and rural residents attending urban

facilities (rural/urban group, $\mathbf{n}=\mathbf{3 7 7}$ )
Multivariate

Poisson

regression

a

Sex (associated, men $<$ women):

significant increased risk to default ART

treatment, HR men: 1.51 [95\% Cl: 1.18-1.93,

$p<0.01]$ Age (not associated): no

significant association between age and

defaulting treatment, compared to younger age ( $\leq 30$ years), $\mathrm{HR}>30$ years: $0.90[95 \% \mathrm{Cl}$ :

$0.70-1.15, p=0.40]$ Severity of disease

associated and WHO stages not associated):

higher CD4 cell count increases significan):

higher CD4 cell count increases significantly risk for defaulting treatment, $\leq 100 \mathrm{CD} 4$ (referent); 101-200 CD4: HR 1.32 [95\% Cl:
$0.99-1.76, p=0.06]$, CD4 > 200 HR: 1.39 [95\% $0.99-1.76, p=0.06], C D 4>200 \mathrm{HR}: 1.39$
Cl 1.02-1.91, $p=0.04]$. No significant

difference in the risk of defaulting treatment being in WHO stage $3 / 4$ or $1 / 2$, HR stage $3 / 4$.

1.14 [95\% Cl: 0.85-1.53, p = 0.37]

Multivariable Sex (not associated): gender is not

Cox associated with risk of LTFU: HR male: 1.1

proportional [95\% Cl: 0.82-3.12, no p value reported, 1.0

hazards falls within $\mathrm{Cl}$ Age (not associated):

regression younger children ( $<2$ yrs) are as likely to

LTFU than older children $(>2$ yrs): $>2$ yrs
Lis:

(referent); HR 1-2 yrs: 1.61 [95\% Cl: 0.96-

2.68, no $\mathrm{p}$ value reported, 1.0 in $\mathrm{Cl}>0.90$ ]

$\mathrm{HR}<1$ yr: $[1.81,95 \% \mathrm{Cl}: 0.94-3.64$, no $p$

value reported, $1.0 \mathrm{in} \mathrm{Cl}$ Severity of

disease (contradicting results, CD4 cell

count not associated and WHO stages

associated): severe clinical status is

associated with risk LTFU: HR severe clinical

status: 1.47 [95\% Cl: 1.03-2.12, no p value

reported, 1.0 not within $\mathrm{Cl}]$ / severe

immunodeficiency was associated with risk LTFU: HR severe immunodeficiency: 0.81

[95\% Cl: 0.52-1.24, p value not reported, 1.0

in Cl Area of living (associated, rural/

urban < urban/urban

$<$ rural/rural): patient in rural areas visiting clinics in urban areas are more likely to

LTFU than patients from rural areas visiting

rural clinics and patients in urban areas

visiting urban clinics: rural (referent); $\mathrm{HR}$

urban: 1.14 [95\% Cl: 0.57-2.24]; HR rural/

urban $2.85[95 \% \mathrm{Cl}, 1.41-5.79][\mathrm{p}=0.004]$ 
Table 4 Overview of findings per study reporting on equity in ART adherence (Continued)

\begin{tabular}{|c|c|c|c|c|c|c|}
\hline $\begin{array}{l}\text { Cornell } \\
2009 * * \\
\text { Observational } \\
{[28]}\end{array}$ & $\begin{array}{l}\text { To investigate the impact of gender } \\
\text { and income on survival and } \\
\text { retention in a South African public } \\
\text { sector ART programme }\end{array}$ & $\begin{array}{l}\text { Urban: Nyanga township, } \\
\text { outskirts of Cape Town, } \\
\text { Western Cape province, } \\
\text { public clinics supported } \\
\text { by NGOs }\end{array}$ & $\begin{array}{l}\text { Sept } 2002 \\
\text { - Apr } 2007\end{array}$ & 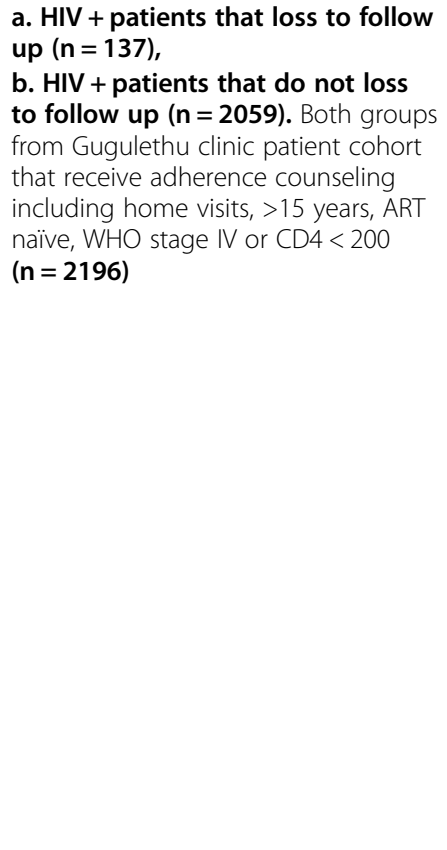 & $\begin{array}{l}\text { Proportional } \\
\text { hazards } \\
\text { regression } \\
\text { models }\end{array}$ & 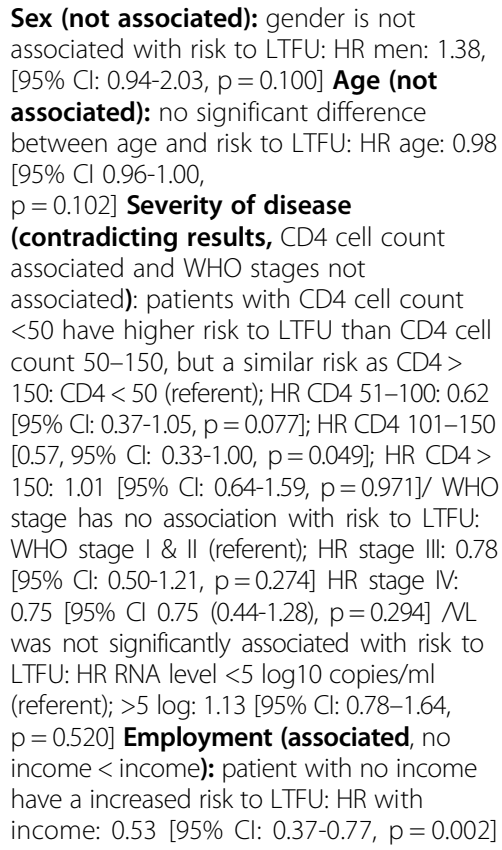 \\
\hline
\end{tabular}


Total score (out of 40 points)

High (30-40 points)

Medium (20-29 points) Low (<20 points)

\section{Studies}

Kranzer Cooke Fatti Govindasamy Boyles Cleary Orrell, Cornell Tsai Adam Muula Nattrass

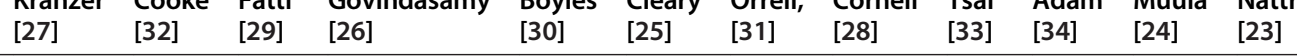

1 Study design (peer reviewed $=2$, other $=0$ )

2 Well-defined hypothesis/objective/research question? (fully $=2$, partial $=1$, not at all =0)

3 Clear motivation research question? (fully $=2$, partial $=1$, not at all $=0$ ) 2

4 Concept clearly defined (e.g. access, equity) (fully $=2$, partial $=1$, not at all 2 $=0)$

5 Methods well described? (fully $=2$, partial $=1$, not at all $=0$ )

6 Main outcomes clearly described? (fully $=2$, partial $=1$, not at all =0) 2

Potential sources of bias taken into account? (fully $=2$, partial $=1$, not at all =0)

8 Population and sampling method clearly defined? (fully $=2$, partial $=1$, not 2 at all $=0$ )

9 Type of information used (i.e. sample size, time period) clearly described? (fully $=2$, partial $=1$, not at all $=0$ )

10 Primary data used for key analyses? (yes $=2$, no $=0$ )

11 Survey (household/provider level) data used? (yes $=2$, partial $=1$, no $=0$ ) 2

12 Research/subquestion(s) answered? (fully = 2, partial = 1, not at all =0)

13 Results based on evidence derived from the data analysis? (fully $=2$, partial $=1$, not at all $=0$ )

14 Results credible given the methods, data, and analysis used? (fully $=2$, partial $=1$, not at all $=0$ )

15 Robustness of findings and limitations of method discussed? (fully $=2$, partial $=1$, not at all $=0$ )

16 Findings discuss within context of existing evidence base? (fully $=2$, partial $=1$, not at all $=0$ )

17 Missings clearly described? (fully $=2$, partial $=1$, not at all $=0$ )

18 Generalizable to rest of the country? (given sample size) (fully $=2$, partial $=1$, not at all $=0$ )

19 Study subjects asked representative of entire population recruited from? (yes $=2, \mathrm{no}=0$ )

20 Study subjects prepared to participate representative of entire population recruited from? (yes $=2$, partial $=1$, no $=0$ ) 
unmarried is associated with lower ART utilization [33]. For ethnicity only one study (high-quality) was found, and it reported inconclusive results due to a small sample size [25]. None of the included studies looked at the ART utilization by religion or sexual orientation.

\section{Discussion}

This is the first systematic review that examines equity in utilization of ART in South Africa and identified 12 studies. It seems that men, young people, those living in certain provinces or rural areas, people who are unemployed or with low educational level, or those who are unmarried have less access to ART. For severity of disease, most studies used more than one outcome measure for disease stage and reported within their study contradicting results. No evidence of inequity in ART utilization by ethnicity, religion and sexual orientation was found. There were large heterogeneities in both context (study area, type of program, time period) and methodology of the studies in this review.

Only one high- and one low-quality study reported a significant difference in utilization of ART among men and women, and eight other studies found no differences. Although it is encouraging that access to ART seems mostly equal for both genders, the studies in our review failed to take the timing of ART initiation into account. Observational studies from South Africa recently showed that case-fatality rates among HIV-infected men were substantially higher compared to women in South Africa, most likely related to late entry into care [35,36]. Late entry by men can be explained as ART is mainly provided through primary health care services, and its antenatal care services frequently serve as an entry- point for HIV treatment for women.

The findings in some studies which showed that young age is associated with low utilization raises concerns. Young people may face more barriers to treatment (like lack of knowledge about treatment possibilities and benefits and fear for stigma and discrimination) [32]. Yet, this relationship may be confounded by eligibility, as older people are more likely to be eligible because of more advanced disease stages. In addition, many studies did not cover all ages. As the HIV epidemic in South Africa is ageing $[37,38]$ it will become increasingly important to determine ART utilization among elderly, a group previously neglected in research on ART utilization.

Both area of living and SES did not seem to be associated with ART utilization. However, the studies looking at area of living were mostly of low-quality. The studies by Nattrass et al. [23] and Adam et al. [34] reported coverage levels for different provinces. However, these studies used a simple Markov-model to estimate the need for ART, and it is difficult to determine whether the model projections are valid. The study by Fatti $e t$ al. [29] reports on children in four different areas. Lower utilization for children living in rural areas and accessing clinics in urban areas can be explained by financial and non-financial barriers such as the monetary cost of transportation or the opportunity cost of accessing health care services [33]. Nevertheless, more research is needed in order to generalize these findings to other areas and population groups. Finally, Tanser et al. [39] showed that self-reported visiting of health clinics in a rural South African area was significantly associated with the distance between the clinic and home, with greater distance resulting in lower utilization, yet we did not include this study because it didn't specifically concern ART utilization.

Studies on SES and area of living will likely measure the same inequities as people in deprived areas might have lower SES. Tsai et al. [33] found significant evidence of socioeconomic inequities in the uptake of ART services within a rural and deprived part of South Africa during the early years of the public sector scaling up of ART (2003-2005). Poorer households in South Africa and in sub-Saharan Africa generally have less access because they face various barriers like cost for transport to the clinic, knowledge of the benefit of ART treatment and a lower propensity to seek formal sector treatment for illness [40,41]. Cleary et al. [25] reported no differences in SES distribution between those in need and those accessing ART in urban areas in 2008. This is in line with the 'inverse equity hypothesis' which predicts a paradoxical worsening of health inequities as effective new public health interventions first diffuse among the well-to-do but later also among the poor. Last years ART has been scaled-up drastically (and now reaches about $80 \%$ of those in need) barriers to access might have been reduced or removed and those least able to overcome those initial barriers are now able to use the services [25]. Yet, still about $20 \%$ lacks access to treatment and this group likely faces most barriers. In addition, if South Africa adopts the new WHO guidelines and further expands its ART program new inequities might appear.

We found contradicting results for severity of disease as within studies differences in ART utilization were reported for HIV-infected people with different CD4 cell count levels but not for different WHO disease stages. Also, some studies reported lower utilization for healthier patients while other studies for the most severely ill. One of the studies by Govindasamy et al. [26] addressed ART initiation and concluded that those with a CD4 cell count of $>350$ are less likely linked to care after testing HIV positive than those $\leq 350$. This can be explained by the fact that these patients were not yet eligible for ART and only needed to enrol in the clinic to monitor their 
CD4 level, or because they feel less need for care as they do not suffer from symptoms. The other five studies addressed ART adherence. Boyles et al. [30] and Kranzer et al. [27] both found that those with higher CD4 cell count (CD4 > 200) adhere less to ART and this may also be explained by the fact that individuals who default do so because they feel better on treatment $[42,43]$. In contrast, Fatti et al. [29] and Orrell et al. [31] found that most severely ill patients were more likely to lost of follow-up. One explanation could be that patients perceived a lack of effectiveness of treatment when ill or not being able to take the medicine because of symptoms [42]. However, the status of patients who are lost to follow-up is difficult to assess, and it is also likely that many of those are unregistered deaths, thus explaining the higher rates among those with advanced disease.

Only one of the studies looked at marital status and reported less access for unmarried people. However, this study was of medium quality as it compared socioeconomic characteristics of a community sample with a clinic sample which were taken from different areas. For ethnicity, religion and sexual orientation no evidence was available and more research is needed to determine inequities in ART utilization by these criteria. It is likely that inequities exist on the basis of ethnicity, as the history of apartheid caused differences in access between black and white South Africans [44]. Also, among black Africans differences in access between ethnic groups like Zulu-speakers, French speaking Cameroonians and Xhosa speakers likely exist, partly due to differences in language barriers that they may face when accessing care $[45,46]$. Although HIV-prevention services for men who have sex with men (MSM) are expanding across the country, there are still several gaps $[47,48]$. This group may face barriers in ART access due to fear of provider stigma and social isolation [49,50]. Low HIV testing rates are reported among Muslim people in predominantly Muslim residential areas in Cape Town [51] and different religions might face different levels of HIV-related stigma which might cause inequities in ART utilization [52].

After analyzing the findings of the included studies we found no patterns of equities or inequities that may be explained by differences in program design (e.g. NGO or university supported, public program, availability adherence counselor), time period (e.g. before or after scale up of ART), target population (e.g. indigent populations, children) and study area (e.g. townships, rural areas). On the other hand, patterns might have been identified if the number of studies included in this review were higher.

We found only 12 studies which looked at equity criteria for ART utilization, and two of these were of low- quality. In addition, all studies differed in context (year of study, area, study population), methodology, and outcome measured. Access to ART in South Africa has evolved quickly over the past decade [53] and inequities that were reported at the start of the ART scale-up might no longer be relevant now. Given the incomplete and mixed evidence base, we call for more rigorous analysis on equity of ART treatment in South Africa, and beyond. We flag three important domains. First, reviewed studies were based on different samples and this made any comparison or generalisation difficult to achieve. A national monitoring system on ART initiation and adherence, which also registers key criteria such as severity of disease, gender, age, SES and area of living could fill in this gap. To measure those in need for ART we recommend using the definition 'eligible for ART on the basis of the country guidelines' as not all HIV-infected people might be already eligible for ART. Yet, the challenge remains to identify HIV-infected patients who are in need of treatment but have not yet been linked to care. Second, most studies only assessed a few equity criteria. This could be explained by the emphasis in strategic ART plans worldwide to reduce gender, SES and area of living inequities [1]. In addition, the recent health equity monitor launched by the WHO uses a list of indicators to present a country's equity profile, but recommends to differentiate groups on the basis of SES, gender, area of living and education level only [54]. We therefore recommend getting similar insights in inequalities between groups that differ in age, severity of disease, marital status, ethnicity, sexual orientation and religion for ART utilization. Third, studies employed a variety of definitions of both ART initiation and adherence measures, but also of equity criteria measures, indicating the need to develop standardized measures in this area of study.

\section{Conclusions}

On the basis of 12 studies identified in this review it seems that men, young people, those living in certain provinces or rural areas, those who are unemployed or with a low educational level, and those who are unmarried are disadvantaged from utilization of ART. For severity of disease, most studies used more than one outcome measure for disease stage and reported within their study contradicting results. For ethnicity, religion and sexual orientation there was no evidence available to draw conclusions. As studies stem from different contexts and use different methods, findings cannot be generalized and conclusions should be taken with caution. In order to better inform policy makers, we call for improved guidance in equity research on ART, addressing the need to develop national monitoring of inequity of utilization of ART and employing standardized measures of utilization and equity criteria. 


\section{Additional files}

\section{Additional file 1: Database search strategies for systematic review} on equity in utilization of ART in South Africa.

Additional file 2: Data extraction and quality assessment forms for systematic review on equity in utilization of ART in South Africa.

\section{Abbreviations}

ART: Antiretroviral therapy; PMTCT: Prevention of mother to child transmission; SES: Socio-economic status; WHO: World Health Organization.

\section{Competing interests}

The authors declare that they have no competing interests.

\section{Authors' contributions}

NT coordinated the study and participated in the design of the study, acquisition, analysis and interpretation of data and helped drafting and finalised the manuscript. CM participated in the design of the study, acquisition, analysis and interpretation of data and drafted the manuscript. EM was involved in the acquisition, analysis and interpretation of the data and revised the manuscript. $J \mathrm{H}$ was involved in interpretation of the data and revised the manuscript. RB was involved in the design of the study, helped in interpretation of the data and revised the manuscript. All authors read and approved the final manuscript.

\section{Author details}

'Department of Primary and Community Care, Radboudumc, Nijmegen, The Netherlands. ${ }^{2}$ Department of Public Health, Erasmus MC, University Medical Center Rotterdam, Rotterdam, The Netherlands.

Received: 26 July 2013 Accepted: 25 June 2014

Published: 1 August 2014

\section{References}

1. UNAIDS: UNAIDS Report on Global AIDS Epidemic 2013. Geneva: 2013 [http:// www.unaids.org/en/media/unaids/contentassets/documents/epidemiology/ 2013/gr2013/UNAIDS_Global_Report_2013_en.pdf]

2. UNAIDS: World AIDS Day Report - Results 2012. Geneva: 2012 [http://www. unaids.org/en/media/unaids/contentassets/documents/epidemiology/2012/ gr2012/JC2434_WorldAIDSday_results_en.pdf]

3. Department of Health Republic of South Africa: The South African Antiretroviral Treatment Guidelines 2013. South Africa: 2013 [http://www. sahivsoc.org/upload/documents/2013\%20ART\%20Guidelines-Short\%20Combined \%20FINAL\%20draft\%20guidelines\%2014\%20March\%202013.pdf]

4. Cleary SM, Mooney GH, Mclntyre DE: Claims on health care: a decision-making framework for equity, with application to treatment for HIV/AIDS in South Africa. Health Policy Plan 2011, 26:464-470.

5. SANAC: South Africa National AIDS Council: National Strategic Plan 2012 2016. South Africa: 2011 [http://www.sanac.org.za/resources/cat_view/2-nsp]

6. Bor J, Herbst AJ, Newell ML, Barnighausen T: Increases in adult life expectancy in rural South Africa: valuing the scale-up of HIV treatment. Science 2013, 339:961-965.

7. Mills EJ, Bakanda C, Birungi J, Mwesigwa R, Chan K, Ford N, Hogg RS, Cooper C: Mortality by baseline CD4 cell count among HIV patients initiating antiretroviral therapy: evidence from a large cohort in Uganda. AIDS 2011, 25:851-855

8. Tanser F, Barnighausen T, Grapsa E, Zaidi J, Newell ML: High coverage of ART associated with decline in risk of HIV acquisition in rural KwaZulu-Natal, South Africa. Science 2013, 339:966-971.

9. Cohen MS, Chen YQ, McCauley M, Gamble T, Hosseinipour MC, Kumarasamy N, Hakim JG, Kumwenda J, Grinsztejn B, Pilotto JH, Godbole SV, Mehendale S, Chariyalertsak S, Santos BR, Mayer KH, Hoffman IF, Eshleman SH, PiwowarManning E, Wang L, Makhema J, Mills LA, de Bruyn G, Sanne I, Eron J, Gallant J, Havlir D, Swindells S, Ribaudo H, Elharrar V, Burns D, et al: Prevention of HIV-1 infection with early antiretroviral therapy. $N$ Engl J Med 2011, 365:493-505.

10. Eaton JW, Johnson LF, Salomon JA, Barnighausen T, Bendavid E, Bershteyn A, Bloom DE, Cambiano V, Fraser C, Hontelez JA, Humair S, Klein DJ, Long EF, Phillips AN, Pretorius C, Stover J, Wenger EA, Williams BG, Hallett TB: HIV treatment as prevention: systematic comparison of mathematical models of the potential impact of antiretroviral therapy on HIV incidence in South Africa. PLoS Med 2012, 9:e1001245.

11. Hontelez JA, de Vlas SJ, Tanser F, Bakker R, Barnighausen T, Newell ML, Baltussen $\mathrm{R}$, Lurie MN: The impact of the new WHO antiretroviral treatment guidelines on HIV epidemic dynamics and cost in South Africa. PLoS One 2011, 6:e21919.

12. Hontelez JA, Lurie MN, Barnighausen T, Bakker R, Baltussen R, Tanser F, Hallett TB, Newell ML, de Vlas SJ: Elimination of HIV in South Africa through expanded access to antiretroviral therapy: a model comparison study. PLoS Med 2013, 10:e1001534.

13. Eaton JWMN, Stover J, Cambiano V, Chindelevitch L, Cori A, Hontelez JAC, Humair S, Kerr CC, Klein DJ, Mishra S, Mitchel KM, Nichols BE, Vickerman P: Health benefits, costs, and cost-effectiveness of earlier eligibility for adult antiretroviral therapy and expanded treatment coverage: a combined analysis of 12 mathematical models. Lancet Glob Health 2014, 2:e23-e34.

14. World Health Organization: Consolidated Guidelines on the Use of Antiretroviral Drugs for Treating and Preventing HIV Infection. Geneva: 2013 [http://apps.who.int/iris/bitstream/10665/85321/1/9789241505727_eng.pdf]

15. Higgins J, Green S: Cochrane Handbook for Systematic Reviews of Interventions Version 5.1.0 [updated March 2011]. Oxford: The Cochrane Collaboration; 2011.

16. World Health Organization: Monitoring Equity in Access to AIDS Treatment Programmes: A Review of Concepts, Models, Methods and Indicators. Geneva: 2010 [http://whqlibdoc.who.int/publications/2010/9789241564120_eng.pdf]

17. Andersen RM: Revisiting the behavioral model and access to medical care: does it matter? J Health Soc Behav 1995, 36:1-10.

18. Starfield B: The hidden inequity in health care. Int J Equity Health 2011 10:15.

19. Tromp N, Baltussen R: Mapping of multiple criteria for priority setting of health interventions: an aid for decision makers. BMC Health Serv Res 2012, $12: 454$.

20. Centre for Reviews and Dissemination: Systematic Reviews: CRD's Guidance for Undertaking Reviews in Health Care. York: 2008 [http://www.york.ac.uk/ inst/crd/pdf/Systematic_Reviews.pdf]

21. Van de Voorde C, Léonard C: Search for Evidence and Critical Appraisal: Health Services Research. Brussels: Belgian Health Care Knowledge Centre (KCE); 2007

22. Moher D, Liberati A, Tetzlaff J, Altman DG, Group P: Preferred reporting items for systematic reviews and meta-analyses: the PRISMA statement. PLoS Med 2009, 6:e1000097.

23. Nattrass N: South Africa's "rollout" of highly active antiretroviral therapy: a critical assessment. J Acquir Immune Defic Syndr 2006, 43:618-623.

24. Muula AS, Ngulube TJ, Siziya S, Makupe CM, Umar E, Prozesky HW Wiysonge CS, Mataya RH: Gender distribution of adult patients on highly active antiretroviral therapy (HAART) in Southern Africa: a systematic review. BMC Public Health 2007, 7.

25. Cleary S, Silal S, Birch S, Carrara H, Pillay-van Wyk V, Rehle T, Schneider H: Equity in the use of antiretroviral treatment in the public health care system in urban South Africa. Health Policy 2011, 99:261-266.

26. Govindasamy D, van Schaik N, Kranzer K, Wood R, Mathews C, Bekker LG: Linkage to HIV care from a mobile testing unit in South Africa by different CD4 count strata. J Acquir Immune Defic Syndr 2011, 58:344-352.

27. Kranzer K, Lewis JJ, Ford N, Zeinecker J, Orrell C, Lawn SD, Bekker LG, Wood R: Treatment interruption in a primary care antiretroviral therapy program in South Africa: cohort analysis of trends and risk factors. J Acquir Immune Defic Syndr 2010, 55:e17-e23.

28. Cornell M, Myer L, Kaplan R, Bekker LG, Wood R: The impact of gender and income on survival and retention in a South African antiretroviral therapy programme. Trop Med Int Health 2009, 14:722-731.

29. Fatti G, Bock P, Grimwood A, Eley B: Increased vulnerability of rural children on antiretroviral therapy attending public health facilities in South Africa: a retrospective cohort study. J Int AIDS Soc 2010, 13:46.

30. Boyles TH, Wilkinson LS, Leisegang R, Maartens G: Factors influencing retention in care after starting antiretroviral therapy in a rural South African programme. PLoS One 2011, 6:e19201.

31. Orrell C, Bangsberg DR, Badri $M$, Wood R: Adherence is not a barrier to successful antiretroviral therapy in South Africa. AIDS 2003, 17:1369-1375.

32. Cooke GS, Tanser FC, Barnighausen TW, Newell ML: Population uptake of antiretroviral treatment through primary care in rural South Africa. BMC Public Health 2010, 10:585. 
33. Tsai AC, Chopra M, Pronyk PM, Martinson NA: Socioeconomic disparities in access to HIV/AIDS treatment programs in resource-limited settings. AIDS Care 2009, 21:59-63.

34. Adam MA, Johnson LF: Estimation of adult antiretroviral treatment coverage in South Africa. S Afr Med J 2009, 99:661-667.

35. Cornell M, Schomaker M, Garone DB, Giddy J, Hoffmann CJ, Lessells R, Maskew M, Prozesky H, Wood R, Johnson LF, Egger M, Boulle A, Myer L: Gender differences in survival among adult patients starting antiretroviral therapy in South Africa: a multicentre cohort study. PLoS Med 2012, 9:e1001304.

36. Druyts E, Dybul M, Kanters S, Nachega J, Birungi J, Ford N, Thorlund K, Negin J, Lester R, Yaya S, Mills EJ: Male sex and the risk of mortality among individuals enrolled in antiretroviral therapy programs in Africa: a systematic review and meta-analysis. AIDS 2013, 27:417-425.

37. Hontelez JA, Lurie MN, Newell ML, Bakker R, Tanser F, Barnighausen T, Baltussen R, de Vlas SJ: Ageing with HIV in South Africa. AIDS 2011, 25:1665-1667.

38. Hontelez JA, de Vlas SJ, Baltussen R, Newell ML, Bakker R, Tanser F, Lurie M, Barnighausen $\mathrm{T}$ : The impact of antiretroviral treatment on the age composition of the HIV epidemic in sub-Saharan Africa. AIDS 2012 26(Suppl 1):S19-S30.

39. Tanser F, Gijsbertsen B, Herbst K: Modelling and understanding primary health care accessibility and utilization in rural South Africa: an exploration using a geographical information system. Soc Sci Med 2006, 63:691-705.

40. Castro-Leal F, Dayton J, Demery L, Mehra K: Public spending on health care in Africa: do the poor benefit? Bull World Health Organ 2000, 78:66-74.

41. Filmer D: Fever and its treatment among the more and less poor in sub-Saharan Africa. Health Policy Plan 2005, 20:337-346.

42. Brinkhof MW, Pujades-Rodriguez M, Egger M: Mortality of patients lost to follow-up in antiretroviral treatment programmes in resource-limited settings: systematic review and meta-analysis. PLoS One 2009, 4:e5790

43. Miller CM, Ketlhapile M, Rybasack-Smith H, Rosen S: Why are antiretroviral treatment patients lost to follow-up? A qualitative study from South Africa. Trop Med Int Health 2010, 15(Suppl 1):48-54.

44. Coovadia H, Jewkes R, Barron P, Sanders D, Mclntyre D: The health and health system of South Africa: historical roots of current public health challenges. Lancet 2009, 374:817-834.

45. Ndlovu M: The Accessibility of Translated Zulu Health Texts: An Investigation of Translation Strategies. University of South Africa; 2009 [http://uir.unisa.ac.za/ bitstream/handle/10500/3400/thesis_ndlovu.pdf? sequence $=1]$

46. Cain D, Schensul S, Mlobeli R: Language choice and sexual communication among Xhosa speakers in Cape Town, South Africa: implications for HIV prevention message development. Health Educ Res 2011, 26:476-488.

47. Mclntyre J, Jobson G, Struthers H, De Swardt G, Rebe K: Rapid Assessment of HN Prevention, Care and Treatment Programming for MSM in South Africa. Assessment report 2013. Johannesburg: Anova Health Institute; 2013 [http://heaids.org.za/site/ assets/files/1227/anova_ejaf_msm_assessment_full_report_v5.pdf]

48. Mclntyre JA, Struthers H: HIV and men who have sex with men in South Africa. AlDS Behav 2013, 17(Suppl 1):S1-S3.

49. Batist E, Brown B, Scheibe A, Baral SD, Bekker LG: Outcomes of a communitybased HIV-prevention pilot programme for township men who have sex with men in Cape Town. South Africa. J Int AIDS Soc 2013, 16:18754.

50. Jobson G, de Swardt G, Rebe K, Struthers H, Mclntyre J: HIV risk and prevention among men who have sex with men (MSM) in peri-urban townships in Cape Town. South Africa. AIDS Behav 2013, 17(Suppl 1):S12-S22.

51. Kagee A, Toefy Y, Simbayi L, Kalichman S: HIV prevalence in three predominantly Muslim residential areas in the Cape Town metropole. S Afr Med J 2005, 95:512-516.

52. Van Breda AD: Stigma as 'othering' among Christian theology students in South Africa. SAHARA J 2012, 9:181-191.

53. UNAIDS: AIDSinfo for South Africa. 2014 [http://www.unaids.org/en/dataanalysis/ datatools/aidsinfo/]

54. World Health Organization: Health Equity Monitor - Compendium of Indicator Definitions. Geneva: 2013 [http://www.who.int/gho/health_equity/ outcomes/health_equity_compendium.pdf]

\section{doi:10.1186/s12939-014-0060-z}

Cite this article as: Tromp et al.: Equity in utilization of antiretroviral therapy for HIV-infected people in South Africa: a systematic review. International Journal for Equity in Health 2014 13:60.

\section{Submit your next manuscript to BioMed Central and take full advantage of:}

- Convenient online submission

- Thorough peer review

- No space constraints or color figure charges

- Immediate publication on acceptance

- Inclusion in PubMed, CAS, Scopus and Google Scholar

- Research which is freely available for redistribution 